\title{
Computer Simulation of Fracture in Aerogels.
}

Brian S. Good

NASA Glenn Research Center

Cleveland, OH 44026, U.S.A.

\begin{abstract}
Aerogels are of interest to the aerospace community primarily for their thermal properties, notably their low thermal conductivities. While the gels are typically fragile, recent advances in the application of conformal polymer layers to these gels has made them potentially useful as lightweight structural materials as well. In this work, we investigate the strength and fracture behavior of silica aerogels using a molecular staticsbased computer simulation technique. The gels' structure is simulated via a Diffusion Limited Cluster Aggregation (DLCA) algorithm, which produces fractal structures representing experimentally observed aggregates of so-called secondary particles, themselves composed of amorphous silica primary particles an order of magnitude smaller. We have performed multi-length-scale simulations of fracture in silica aerogels, in which the interaction between two secondary particles is assumed to be described by a Morse pair potential parameterized such that the potential range is much smaller than the secondary particle size. These Morse parameters are obtained by atomistic simulation of models of the experimentally-observed amorphous silica "bridges," with the fracture behavior of these bridges modeled via molecular statics using a Morse/Coulomb potential for silica. We consider the energetics of the fracture, and compare qualitative features of low-and high-density gel fracture.
\end{abstract}

\section{INTRODUCTION}

Aerogels are low-density materials possessing properties that have maintained interest for a wide variety of applications [1-3]. In particular, the low thermal conductivities characteristic of such gels have led to the aerospace community's interest in these materials as lightweight thermal insulation.

Aerogels are also notoriously fragile, and to develop thermal insulation that is mechanically robust, researchers in our laboratory have developed a method for applying conformal polymer coatings aerogels that greatly improve the gels' strength while minimally impacting their insulating properties [4]. In order to provide a microscopic understanding of the mechanical behavior of the gels, and to provide a predictive tool of use in their further development, we have constructed a multiscale model for fracture in silica aerogels. The model is built on computer simulations using a modified diffusionlimited cluster aggregation (DLCA) scheme [5] for gel cluster structure, and an interparticle interaction potential based on results of atomistic simulations.

\section{STRUCTURAL MODEL}

Silica aerogels are known to exhibit a low-density pearl-necklace structure that consists of tangled strands of roughly spherical "secondary" particles. These particles 
themselves exhibit complex internal structure, and are usually considered to consist of smaller "primary" particles of amorphous silica of less than bulk density. For the current work, we ignore this small-scale structure and assume that the secondary particles are of a uniform density less than that of bulk amorphous silica, $\rho=2.20 \mathrm{~g} / \mathrm{cm}^{3}[6]$; an in-chain density of about $1.8 \mathrm{~g} / \mathrm{cm}^{3}$ has been reported by Woignier et $\alpha$ [ [7]. X-ray and neutron probes of gel structure[8-11], suggest that the gels consist of disordered aggregates of connected fractal clusters, with fractal behavior evident over a limited range of length scales.

Several computer simulation techniques have been developed with the goal of producing aggregates that mimic the structures of low-density compact or fractal materials, notably the Eden model [12], ballistic aggregation [13], diffusion-limited aggregation (DLA) [14], and diffusion-limited cluster aggregation (DLCA) [5].

In the current work, we make use of a modified version of the off-lattice DLCA model. Briefly, the DLCA model consists of a number of particles, initially distributed at random without overlap through a computational cell. Particle sizes may be uniform, or chosen randomly from a distribution, as in our work, where a log-normal distribution is used. A particle (or a cluster of them, as the aggregation process proceeds) is chosen at random, with a probability given by $P_{i}=\left(m_{i} / m_{0}\right)^{\alpha}$, where $m_{i}$ and $m_{0}$ are the masses of the moving cluster and the lightest cluster in the cell, respectively, and $\alpha$ is a scaling parameter, here taken to be -0.5 . The particle or cluster is moved in a random direction, and is then inspected for collisions with other particles or clusters. If one or more collisions occur, the colliding clusters and particles are merged into a single cluster. The move-merge procedure is repeated until all particles have merged into a large single cluster.

\section{MULTISCALE FRACTURE MODEL}

The DLCA simulations described above provide a model of the structure of the cluster of secondary particles that make up the gel, and these model clusters exhibit the low-density chain structure characteristic of real gels. Based on examination of micrographs, we assume that the chains consist of secondary particles connected by cylindrical bridges whose diameter is typically smaller than those of the connected particles. Failure is assumed to occur through the breaking of the bridges, with the particles themselves remaining intact.

To describe the details of fracture, we use a multiscale model, in which interparticle interactions are described via a simple interaction potential whose parameters are obtained from a higher-fidelity atomistic simulation of the energetics of bridge strain. Bridge strain energetics are obtained as follows. A cylindrical computational cell of amorphous silica is constructed, with slabs at the top and bottom of the cell (each consisting of 20 percent of the cell vertical dimension) held atomically rigid. The cell is strained by applying a small displacement to the top slab, and the center 60 percent of the cell is allowed to relax via a molecular statics procedure, in which each atom is moved along the local energy gradient. Interatomic interactions include Morse and Coulomb contributions, with fixed partial atomic charges. Potential parameters are shown in Table 1.

$$
V(r)=V_{0}\left[\exp \left(\gamma r_{i j} / r_{0}\right)-2 \exp \left(r_{i j} / 2 r_{0}\right)\right]+q_{i} q_{j} / r
$$


Table 1. Potential parameters

\begin{tabular}{|l|l|l|l|}
\hline & $\mathrm{V}_{0}$ ergs & $\mathrm{r}_{0}, \mathrm{~cm}$ & $\gamma$ \\
\hline $\mathrm{Si}-\mathrm{Si}$ & $2.0537 \mathrm{E}-14$ & $3.4103 \mathrm{E}-8$ & 11.7139 \\
\hline $\mathrm{Si}-\mathrm{O}$ & $3.1957 \mathrm{E}-12$ & $1.6148 \mathrm{E}-8$ & 8.8022 \\
\hline $\mathrm{O}-\mathrm{O}$ & $3.7260 \mathrm{E}-14$ & $3.7835 \mathrm{E}-8$ & 10.4112 \\
\hline
\end{tabular}

$\mathrm{q}_{\mathrm{si}}=1.22 \mathrm{e} ; \mathrm{q}_{\mathrm{o}}=0.61 \mathrm{e}$

To describe the behavior of the gel cluster, we fit the bridge energy versus strain curve to a Morse potential of the same form as the first term in Equation 1. Note, however, that in an atomistic context, the range of the potential is comparable to the size of the interacting atoms; as used to describe secondary particle interactions, however, the potential's range is much smaller than the typical secondary particle diameter.

\section{RESULTS AND DISCUSSION}

We have performed simulations for a range of initial densities (assuming secondary particle density equal to the reported in-chain density), including three which we have characterized as low $\left(0.0130 \mathrm{~g} / \mathrm{cm}^{3}\right)$, medium $\left(0.0603 \mathrm{~g} / \mathrm{cm}^{3}\right)$ and high $\left(0.593 \mathrm{~g} / \mathrm{cm}^{3}\right)$. These densities bracket the observed densities of actual silica gels; for example, Rousset et al. report SEM observations on gels having densities of $0.107,0.23$, and $0.36 \mathrm{~g} / \mathrm{cm}^{3}[3]$. Micrographs of two coated aerogels of different densities are shown in Figure 1.
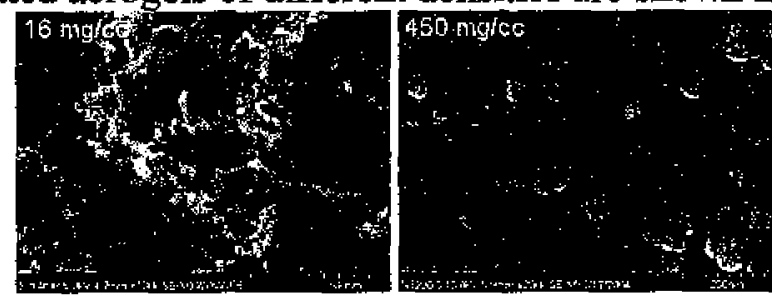

Figure 1. Silica aerogels. $0.016 \mathrm{~g} / \mathrm{cm}^{3}$ (left), $0.450 \mathrm{~g} / \mathrm{cm}^{3}$ (right).

Illustrations of the final states of low, medium and high-density computational cells are presented in Figures 2 (a-c). Fractal dimensions of the clusters are computed from scaling exponent of the cluster mass. The high-density cluster yields a fractal dimension of 2.8 , close to the value of 3 exhibited by compact clusters. The medium- and lowdensity clusters show an inflection point in the $\ln (\mathrm{M}(\mathrm{r}))$ versus $\ln (\mathrm{r})$ curve suggestive of a fractal-to-compact transition; both clusters show a fractal dimension of 2.7-2.9 at large r, with values of 2.0 (medium density) and 1.8 (low density) at small $\mathrm{r}$. The small-r value for the low-density cluster is consistent with the fractal dimension of about 1.75 reported for other colloidal materials [15] 


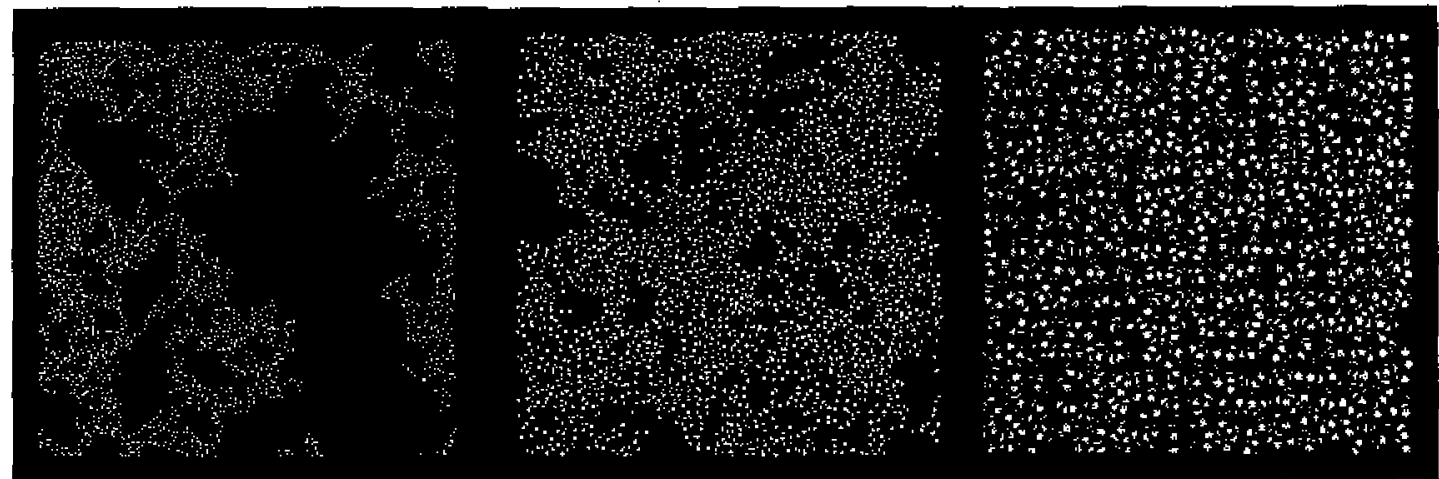

Figure 2. Aerogel clusters from DLCA simulations. (a) $0.0130 / \mathrm{cm}^{3}$ (b) $0.0603 \mathrm{~g} / \mathrm{cm}^{3}$ (c) $0.593 \mathrm{~g} / \mathrm{cm}^{3}$.

Bridge strain energetics are shown in Figure 3. The curve of energy versus strain is typical of phenomena consistent with the Universal Binding Energy Relation (UBER) of Rose, Ferrante and Smith [16]. Such interactions are typical able to be fit reasonably well with a Morse potential, as is done here.

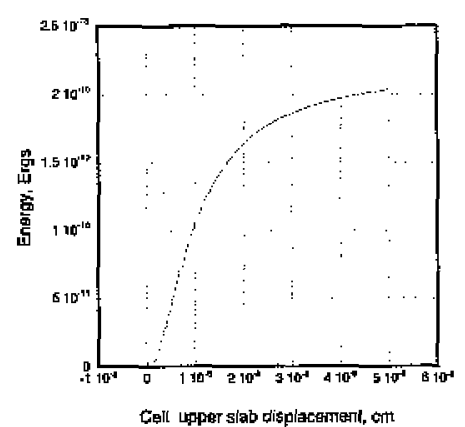

Figure 3. Bridge strain energy

With secondary particle interactions described by this Morse potential, a tensile strain is applied to a series of low-, medium- and high-density DLCA clusters, using the same molecular statics procedure described above. The evolution of a typical highdensity computational cell is shown in Figure 4. Energies as a function of strain for low-, medium- and high-density clusters (averaged over 10,10 and 9 cells, respectively) are shown in Figure 5. 


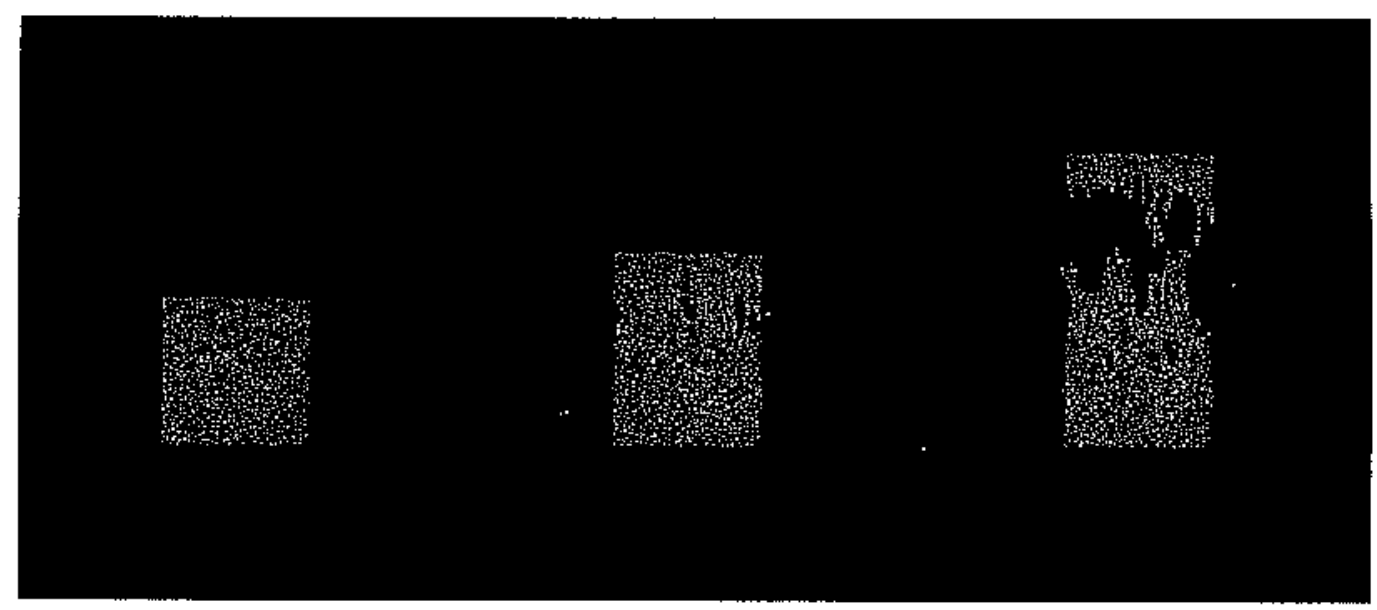

Figure 4. Evolution of failure of high-density cluster

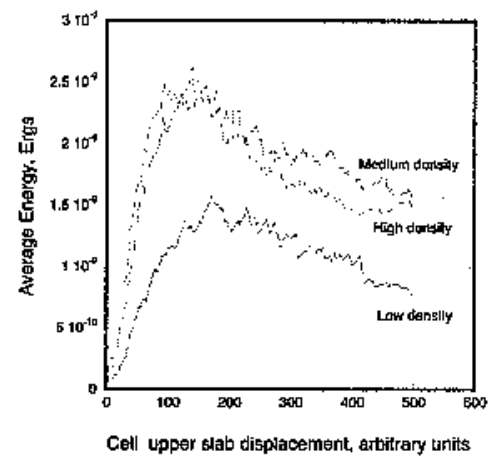

Figure 5. Cluster average energy versus strain

Aerogel fracture is generally considered to be brittle, though the application of such terminology to a gel material may be inappropriate. Regardless, brittle fracture is typically characterized by a lack of necking of a sample fractured in tension, and by an increase in energy with strain, followed by an abrupt decrease in energy when fracture occurs. It is evident from Figure 4 that little or no necking is in evidence. However; the energy versus strain curves in Figure 5 are more ambiguous. The high-density cells appear to exhibit a reasonably abrupt decrease in energy. The medium- and low-density cells show a less abrupt decrease, and it is not clear whether it is appropriate to characterize the failure there as brittle. We are currently awaiting experimental results which we expect to resolve this issue. Because the DLCA clusters at a given density differ slightly in size, an exact calculation of the strain at which failure occurs, based on 
energies averaged over a number of cells of different size, is of limited usefulness.

However, failure occurs at between one and two percent strain for all cluster densities.

A possible explanation for the strain energy behavior is suggested by examining the final stage in the evolution of the computational cell in Figure 4. It appears that some of the "pearl necklace" strands have uncoiled and stretched in the direction of the applied strain, and this uncoiling may tend to make the energy decrease at failure less abrupt. We suspect that this uncoiling may be a consequence of the spherical symmetry of the secondary particle interaction potential; in our model, there is no energy penalty involved when a particle slides across the surface of another. In reality, such motion would involve at least a good deal of chemical bond strain, and, for a significant displacement, the breaking and possible reforming of a number of chemical bonds. For this reason, we are modifying our model to include an angular energy contribution, to be computed atomistically in the same manner as the bond strain energy used here. 


\section{CONCLUSIONS}

We have performed computer simulations of the fracture of silica aerogels, based on a diffusion-limited cluster aggregation model for the gel structure, and a multiscale model for fracture. The simulations were carried out at levels of density that span the range of densities exhibited by real aerogels, with cluster fractal dimensions that are increasing functions of density, and range from 1.9 to 2.9. Model gel fracture occurs at strains of approximately one percent. Strain energetics suggest that the model may not fully capture the brittle behavior exhibited by real gels, and modifications to the particle interaction potential are under way to enhance the fidelity of the simulations.

\section{REFERENCES}

1. N. Husing and U. Schubert, Angew. Chem. Int. Ed. 37, 22 (1998).

2. A. C. Pierre and G. M. Pajonk, Chem. Rev. 2002, 102, 4243 (2002).

3. J. L. Rousset, A. Boukenter, B. Champagnon, J. Dumas, E. Duval, J, F, Quinson and J. Serughetti, J. Phys.: Condens. Matter 2, 8445 (1990).

4. M. A. B. Meador, E. F. Fabrizio, F. Ilhan, A. Dass, G. Zhang, P. Vassilaras, J. C. Johnston and N. Leventis, Chem. Mater. 17, 1085 (2005).

5. P. Meakin, Phys. Rev. Lett. 51, 1119 (1983).

6. O. V. Mazurin, M. V. Streltsina and T. P. Shvaiko-Shvaikovskaya, 1983 Handbook of Glass Data, Part A: Silica Glass and Binary Silicate Glasses, Elsevier, Amsterdam.

7. Woignier : T. Woignier and J. Phalippou, J. Non-Cryst. Solids 9317 (1987).

8. D. W. Schaeffer, J. E. Martin and K. D. Keefer, Phys. Rev. Lett. 56, 2199 (1986).

9. T. Freltoft, J. K. Kjems and S. K. Sinha, Phys. Rev. B 33, 269 (1986).

10. R. Vacher, T Woignier, J. Pelous and E. Courtens, Phys. Rev. B 37, 6500 (1988).

11. A. Hasmy, E. Anglaret, M. Foret, J. Pelous and R. Julien, Phys. Rev. B 50, 1305 (1994).

12. M. Eden in Proc. Of the Fourth Berkeley Symp. Of Mathematical Statistics and Probability, Vol. IV, F. Neyman, ed., University of California, Berkeley, 1961.

13. D. Bensimon, B. Sharaiman and S. Liang, Phys. Lett. 102A, 238 (1984).

14. T. A. Witten and L. M. Sander, Phys. Rev. Lett. 47, 1400 (1981).

15. D. A. Weitz and M. Oliveria Phys. Rev. Lett. 52, 1433 (1984).

16 J. H. Rose, J. Ferrante and J. R. Smith, Phys. Rev. Lett. 47, 675 (1981). 